\title{
A Distributed System Model for Managing Data Ingestion in a Wireless Sensor Network
}

\author{
Washington Velásquez Vargas, Andres Munoz-Arcentales
}

\author{
Joaquín Salvachúa Rodríguez
}

\begin{abstract}
In this paper, a distributed communication model is proposed to be implemented as part of a reactive evacuation management system. This implementation includes elements of wired and wireless network communications, which are used for sending and receiving information about the environment inside of a building. In this type of systems, where the sensor nodes of the network are continuously communicating, it presents a challenge for the classification and processing of the information. The aim behind this design, is using Zookeeper topics in order to allow Kafka server for transmitting the information of an alert state, this technique allow for the system to manage real time data transmission coming from the sensor nodes. Moreover, with this data, we can indicate an evacuation route through the reactive activation signaling. As this is an on-going study, we only illustrate our implementation with structural diagrams, but exclude the performance evaluation results in this paper.

Keywords-Kafka, wireless sensor networks, reactive signaling, algorithms, broadcast messages
\end{abstract}

\section{INTRODUCTION}

The implementation of evacuation systems is required on public places due to the amount of people that they receive every day and who need to be protected in an emergency situation. In this regard, public places have to manage an efficient communication structure module which allow to know the optimal routes to take for evacuation instead of those routes which are congested. [1]

Currently, the enterprises want to adapt big data techniques, such as Hadoop ${ }^{1}$ or Spark $^{2}$ to their traditional tools. In recent years, some projects, such as, connected smart factory, industry 4.0 [2] and factory of the future [3] has been emerged to develop adaptive infrastructures, such as cyber physical systems (CPS) [4], Industrial internet of things (ПIoT) [5] or proposals like the presented by Zafar Iqbal, Kiseon Kim and Heung-No Lee about a cooperation scheme for (Wireless Sensor Networks) WSNs [6], They propose consists, in a network of small cooperative groups of sensors, the nodes help relay information for its neighbor nodes with a significant reduction in energy consumption at the cost of an acceptable reduced throughput and so on. Hence, the motivation of this projects is improving the capability data processing and analysis as well as maintaining the existing systems.

\footnotetext{
${ }^{1}$ Apache Hadoop: http://hadoop.apache.org/

${ }^{2}$ Apache Spark. http://spark.apache.org/
}

In this work, a new approach based on the design of a distributed communication network is proposed using Kafka cluster and big data techniques of streaming processing and data integration [7] for emergency evacuation of ESPOL's Administrative building $\left(1692 \mathrm{~m}^{2}\right)$. The aim is focusing on developing a system to gather and store high-velocity stream data from a wireless sensor network (WSN) at the building with a scalable way [8], [9]. The data processed by sensor nodes, the base station and server are used to graph the evacuation flow, which allows to minimize evacuation time and safeguard the person's life. Each component of the evacuation system operates together to manage the congestion at every circumstance during a disaster event.

The present paper is divided in three sections: Section $I$ describes the components of the model. Section III presents the communication design. Finally, the paper presents the conclusions about of communication model for evacuation systems and its potential benefits.

\section{MOdel COMPONENTS}

The model is based on four stages: data acquisition by the sensors, data flow, data processing and activation of signaling.

\section{A. Data acquisition}

Sensor nodes manage an unique communication interface in 802.15.4 standard and are responsible for sending information about carbon monoxide (CO), carbon dioxide (CO2), the petroleum liquid gas (PLG), vertical liquid level (VLL) and temperature (TMP). The nodes are distributed within the Administrative building, covering all entry points of the blocks in it. In addition to monitor the environmental conditions, a component adds robustness to the evacuation system by applying detection techniques and analyzing the human behavior patterns [10] through video cameras (VC). The video detection component interacts with the route determination process and signaling activation in order to display the optimal route for evacuation. The implementation of this component is out of the scope of this paper.

\section{B. Data flow}

A distribution node has been designed, it has three communication interfaces, the Ethernet interface is used to com- 


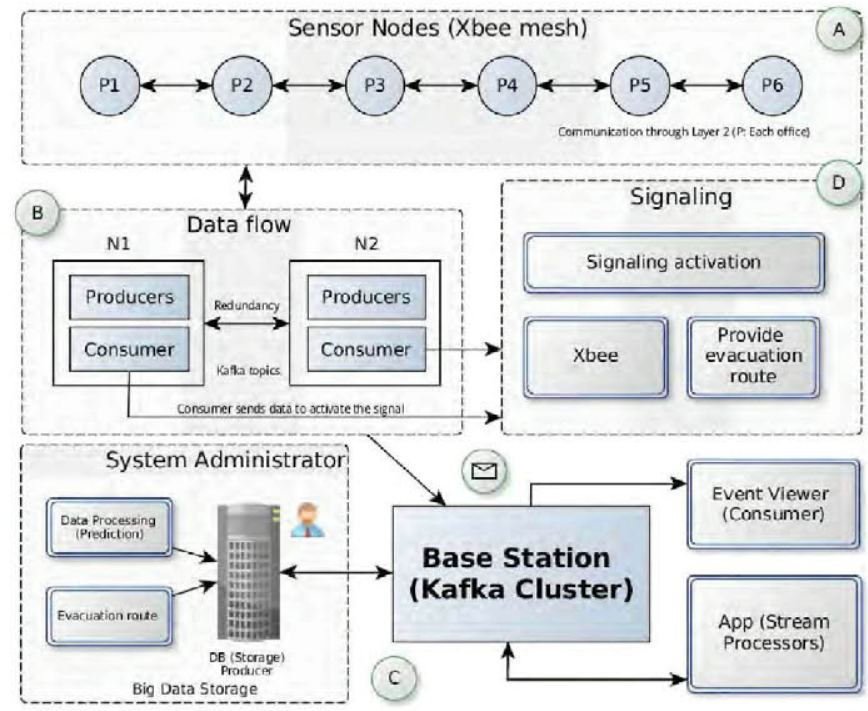

Fig. 1. Architecture of a wireless sensor network with a centralized cluster

municate data with $\mathrm{Kafka}^{3}$ cluster. However, a 3G wireless mobile communication link has been considered as backup, if the Ethernet interface is down. It guarantees the proper functioning of the evacuation system even when the communication interface can be affected for an emergency situation and a Zigbee ${ }^{4}$ module for the communication with the WSN. In Fig. 1, section B shows two nodes that will be communicated to each other to provide redundancy in the transmission (N1: Principal Node, N2: Backup Node).

\section{N1 : Principal Node (Data Flow)}

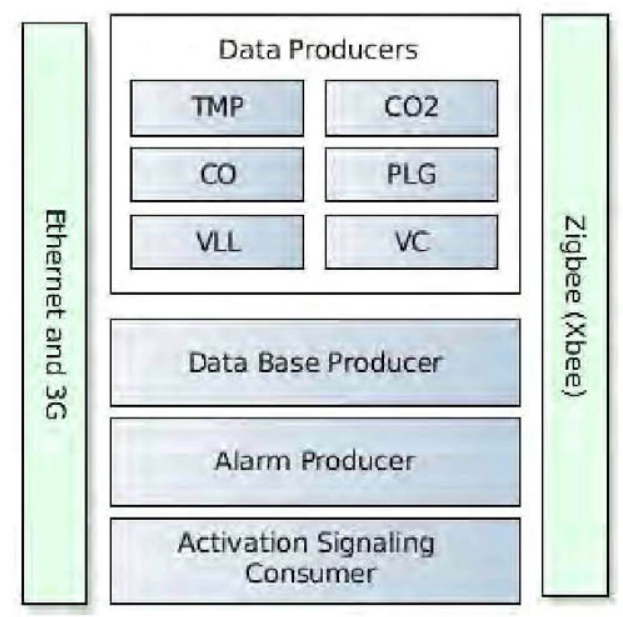

Fig. 2. Communication node between the WSN mesh and the kafka cluster

The Fig. 2 shows the producers and consumers who are sending and receiving data, as follows:

\footnotetext{
${ }^{3}$ Apache Kafka: https://kafka.apache.org/

${ }^{4}$ http://www.zigbee.org/
}

- Data Producers: Responsible for data information from the WSN nodes and transmitting it to the producers of the Kafka cluster.

- Data Base Producer: This producer is in charge to transmit the data producers information to the database, because the apps (stream processors) will use information from the data producers and we want to avoid overloading.

- Alarm Producer: When data are obtained from the sensors, the node processes them to determine if there are any drawbacks with the thresholds that have been configured for each metric. If there were any anomalies, an "Alarm" packet is sent in the format specified in figure 4.

- Activating Signaling Consumer: This consumer will be in charge of processing the information sent by the server for the signaling activation (see Section II D).

\begin{tabular}{|l|l|l|l|}
\hline ID Node & MAC Xbee & Sensed value & Checksum \\
\hline
\end{tabular}

Fig. 3. Package design sent by data producers to kafka cluster

The Fig. 3 shows the frame that is sent by each data producer and it includes the following fields:

- ID Node: Identifier of the node that sends this information

- MAC Xbee: MAC of each of the modules Xbee.

- Sensed value: This is the value sensed by each of the sensors in the mesh, this value will depend on the sensor.

- Checksum: Responsible for validating the frame.

\begin{tabular}{|l|l|l|}
\hline ID Node & Status & Checksum \\
\hline
\end{tabular}

Fig. 4. Package design sent by producers when there is an emergency

The Fig. 4 shows the frame that is sent by the alarm producer which includes the following fields:

- ID Node: Node identifier with some problem

- Status: A status value is sent to identify the alarm priority (0: low, 1: medium, 2: high)

- Checksum: Responsible for validating the frame.

\section{Data processing}

The data are processed by a server that receives messages from nodes through the Kafka cluster, so if one of more sensors which operate with nodes detect any incidence, they will automatically send a message thought the data producers to base station informing about the problem. Then, the base station is responsible for extracting the most relevant information from the message and add the corresponding headers to be sent to the server using UDP protocol or using a mobile network. Once the information arrives to the server, it will start running the algorithm to determine the best evacuation route in each router node which is associated with the reactive signaling. 
Moreover, data producers provide an interactive visualization interface (this is still in development stage) to be used by relief agencies. This interface is being simulated using a virtual modeling technology based on threejs ${ }^{5}$. This tool allows us to model very precisely the infrastructure in buildings and also visually displays the distribution of evacuation routes generated when an emergency occurs (event viewer). The aim is to provide to relief organizations a system that allows them to visualize the unexpected emergency in real time and accurately, so that they can take immediate and coordinate actions based on how the incident evolves. The implementation of this component is out of the scope of this paper.

\section{Signaling Activation}

The algorithm for detecting an evacuation route is based on different stages of interaction between all components of the communications network and previous analysis of management traffic modeling systems evacuation [11], [12], [13]. The reactive signaling component shows to the user the optimal and fastest route to take in emergency situations through the visual signals and the activation of light sequence. This component consists of a small device which includes a micro-controller, RGB LED strips and a Radio Frequency (RF) communication.

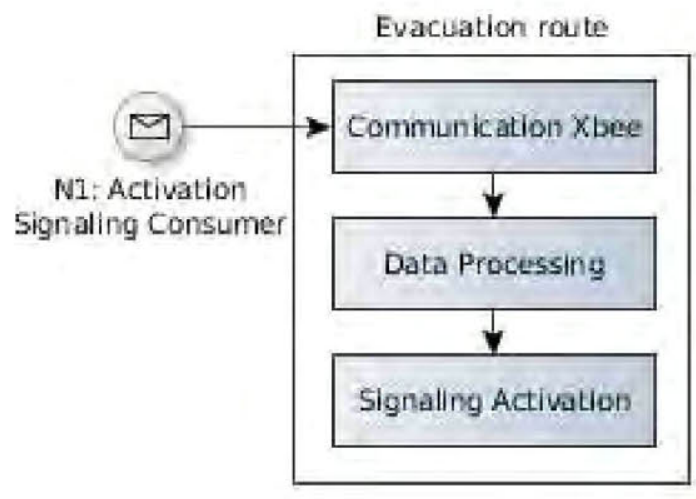

Fig. 5. Signal Activation Process

In Fig. 5, once the micro-controller receives a frame of emergency through "activation signaling consumer" over the RF module, it activates a sequence of lights. Green lights display the optimal route to be followed during the evacuation while red lights indicate that the route is blocked or disabled. Therefore, the reactive signaling component interacts directly with the users by showing them the best route to take in an emergency situation. The signaling receives the frame and automatically turn on the lights in the nearest direction to the evacuation path.

The Fig. 6 shows the frame processed by the microcontroller which includes the following fields:

- ID Signal: Signaling identifier which is switched on in the case of loss or disaster.

${ }^{5}$ https://threejs.org/

\begin{tabular}{|l|l|l|l|}
\hline ID Signal & Status & Address & Checksum \\
\hline
\end{tabular}

Fig. 6. Package design sent by producers when there is an emergency

- Status: This value is used to define the level of emergency "Low, Medium and High" and the color scheme to be showed to users during the evacuation.

- Address: Two bits are used to indicate the direction where signaling has to point out (10 - Left 01 - Right)

- Checksum: Responsible for validating the frame.

\section{Communication DESIGN}

In this section, we introduce the Kafka topics of the wireless sensor network collected from sensor nodes in the first building floor to a centralized clustered server.

The sensor data are classified by type of information being transmitted, each of the metrics has a specific topic and a specific replica for sending packets, e.g. If a sensor node sends temperature values, it will send the information to Two topics (1: Topic TMP and 2: Replica TMP). In the Kafka cluster the Zookeeper topics have been declared in: carbon monoxide (CO), carbon dioxide (CO2), the petroleum liquid gas (PLG), vertical liquid level (VLL), temperature (TMP), video camera (VC) and replicas for each one, as shown in figure 7.

The design is based on the different applications that will have access to the servers, among them the event viewer, all of these will have permission to read the information of the Topics and the Replicas will be in charge of transmitting the information to storage of big data. In addition, a database management topic has been implemented for interaction with big data techniques, this will aid in data processing without interfering with the process of the applications.

In the model there are two topics in particular that help the activation of signaling:

- Alarm (AL): This topic receives the alarms produced by the sensor nodes and re-transmits them to the server to verify the type of event.

- Signal (SN): If an alarm occurs the server sends a signaling activation packet to this topic, then the consumer of the distributed nodes sends the information to activate the signals.

Finally, the architecture presented has the main goal to classify and provide the redundancy to the data gathered, leading to the server only the task of processing it, this reduces the information processing time, as a result of that, the system provides immediate response in the visualization of the evacuation routes inside the affected building.

\section{Conclusions}

In this paper a distributed communication model which provides an as complement of a reactive evacuation system is proposed. The model presented allow to establish a convergence between the devices used by the system and 


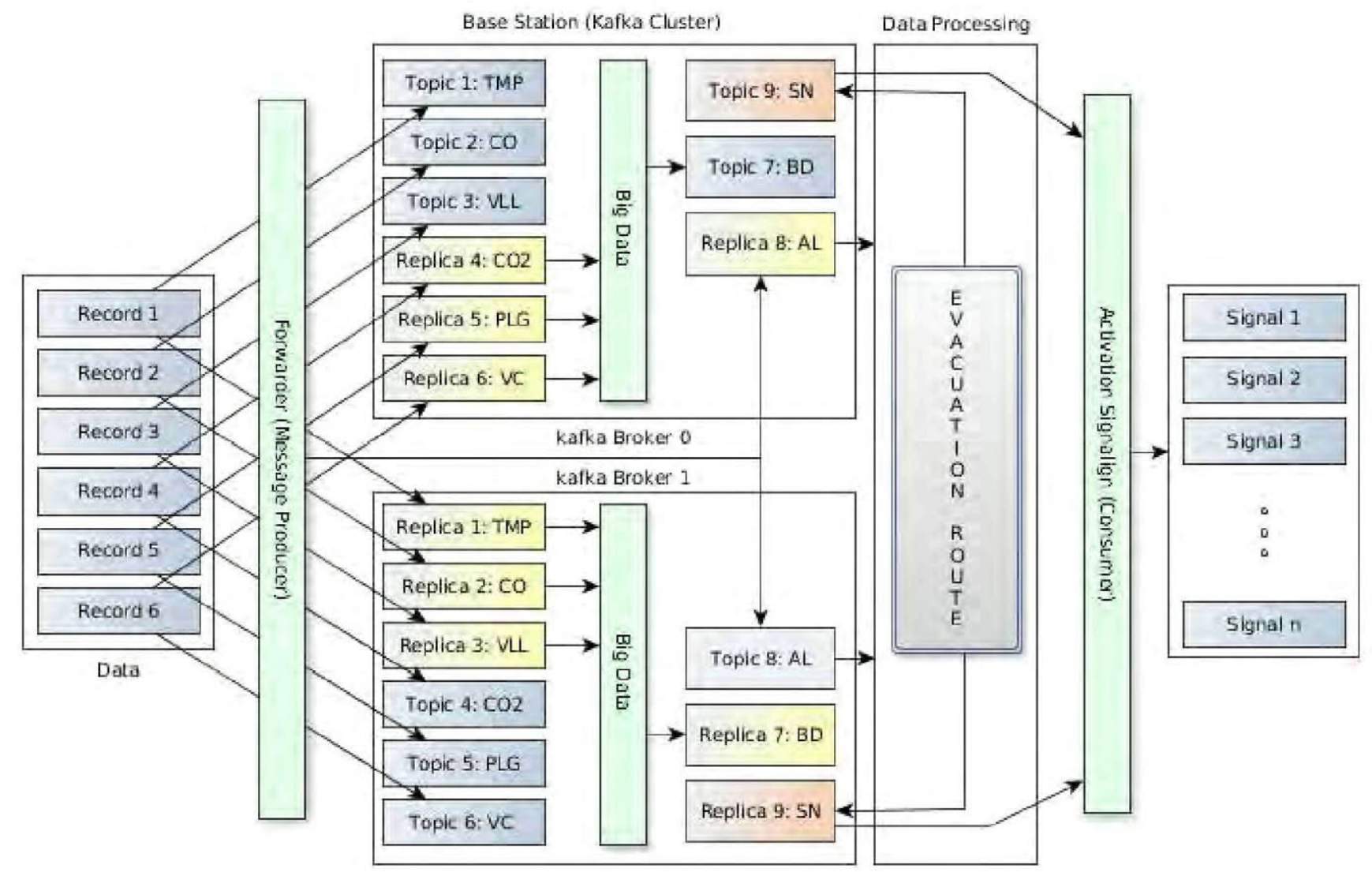

Fig. 7. Distributed architecture using a Kafka cluster

the signaling component through the Kafka server as core distribution.

This design is presented as a basis for the integration or improvement of processes within existing emergency evacuation systems, considering factors such as data integrity, execution speed, concurrency and reliability. Using data segmentation, the system ensures that Alarm signals are processed independently with a specific topic, saving time, in this way that the server processing only has to be in charge of the data processing to generate an optimal evacuation route in the shortest possible time.

The model proposed foresees a more in-depth future study of the real-time processing of the data, due to, a topic has been specified for each of the metrics that are being measured at the building, the intention of this is making use of Spark Streaming and additional and functional programming with Akka in each one of the devices that will be listening the signaling activation, allowing to save on processing and memory used in each one of them.

As this is an ongoing study, we will try to exploit data techniques to predict possible emergencies and safeguard lives. From this, is necessary to decide what storage standard to save and how long it will remain active in the database without harming the performance of the system.

\section{ACKNOWLEDGMENT}

Thanks to the Escuela Superior Politécica del Litoral (ESPOL) and to the Secretaría Nacional de Educación Superior, Ciencia, Tecnología e Innovación (SENESCYT) for financing the continuity of reactive evacuation systems study in emergencies.

\section{REFERENCES}

[1] J. A. Munoz, V. Calero, I. Marin, P. Chavez, and R. Perez, "Adaptive evacuation management system based on monitoring techniques," Latin America Transactions, IEEE (Revista IEEE America Latina), vol. 13, no. 11, pp. 3621-3626, 2015.

[2] W. W. Kagermann, H. and J. Helbig, "Recommendations for implementing the strategic initiative Industrie 4.0: Final report of the Industrie 4.0 Working Group." http://www.acatech.de/fileadmin/user upload/Baumstruktur_nach_Website/Acatech/root/de/Material_fuer_ Sonderseiten/Industrie_4.0/Final_report_Industrie_4.0_accessible.pdf, 2013. [Online]

[3] "Factory of the future." http://ec.europa.eu/research/industrial_ technologies/factories-of-the-future_en.html. [Online].

[4] S. K. Khaitan and J. D. McCalley, "Design techniques and applications of cyberphysical systems: A survey," IEEE Systems Journal, vol. 9, no. 2, pp. $350-365,2015$.

[5] "Accenture. Labs Insight Industrial Internet of Thigs." https://www. accenture.com/us-en/labs-insight-industrial-internet-of-things. [Online].

[6] Z. Iqbal, K. Kim, and H.-N. Lee, "A cooperative wireless sensor network for indoor industrial monitoring," IEEE Transactions on Industrial Informatics, 2016. 
[7] J. Kreps, I Heart Logs: Event Data, Stream Processing, and Data Integration. O'Reilly Media, Inc, first ed., September 2014.

[8] S. Newman, Building Microservices - Designing Fine Grained Systems. Packt Publishing Ltd., first ed., December 2014.

[9] P. R. Nicolas, Scala for Machine Learning - Leverage Scala and Machine Learning to construct and study systems that can learn from data. Mike Loukides and Brian MacDonald, first ed., February 2015.

[10] T. Chen and S. Lu, "Object-level motion detection from moving cameras," IEEE Transactions on Circuits and Systems for Video Technology, vol. PP, no. 99, pp. 1-1, 2016.

[11] A. Desmet and E. Gelenbe, "Reactive and proactive congestion management for emergency building evacuation.,"

[12] A. J. Pel, M. C. Bliemer, and S. P. Hoogendoorn, "A review on travel behaviour modelling in dynamic traffic simulation models for evacuations," Transportation, vol. 39, no. 1, pp. 97-123, 2012

[13] S. Escolar, D. Villa, F. J. Villanueva, R. Cantarero, and J. C. Lpez "An adaptive emergency protocol for people evacuation in high-rise buildings," in 2016 IEEE Symposium on Computers and Communication (ISCC), pp. $364-371$, June 2016. 